\title{
Systemic therapies for unresectable locoregional melanoma: a significant area of need
}

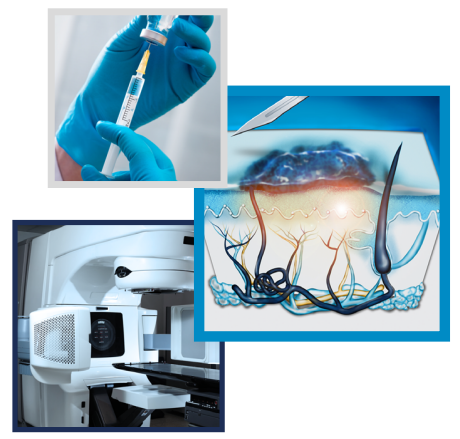

\author{
Emilia Nan Tie ${ }^{1}$ (D), Julia E Lai-Kwon² \& David E Gyorki*,1,3,4 \\ ${ }^{1}$ Division of Cancer Surgery, Peter MacCallum Cancer Centre, Melbourne, VIC, Australia \\ ${ }^{2}$ Department of Medical Oncology, Peter MacCallum Cancer Centre, Melbourne, VIC, Australia \\ ${ }^{3}$ Department of Surgery, University of Melbourne, Melbourne, VIC, Australia \\ ${ }^{4}$ Melanoma and Skin Cancers, Victorian Comprehensive Cancer Centre, Melbourne, VIC, Australia \\ *Author for correspondence: Tel.: +61 038559 7666; David.Gyorki@petermac.org
}

\section{Practice points}

- Immunotherapy and targeted therapy produce sustained improvements in overall survival in metastatic melanoma.

- However, unresectable stage III disease is under-represented in seminal trials of novel therapies. Therefore, the application of this data to patients with unresectable stage III melanoma is unclear.

- Unresectable stage III disease may be technically or clinically unresectable.

- Prospective studies should consider prespecifying subgroups of unresectable disease for analysis.

- Future directions include comparing systemic therapy with and in combination with locoregional therapies.

Immune checkpoint inhibitors and BRAF-MEK inhibitors have revolutionized the management and prognosis of patients with metastatic melanoma. However, there is minimal evidence to guide their incorporation into current treatment paradigms for unresectable stage III disease. The era of effective systemic therapies has prompted a discussion about what constitutes unresectable disease. Patients with unresectable stage III disease can experience significant morbidity from their disease and locoregional therapies, and may progress with distant metastases. Despite increasing use of systemic therapies in unresectable stage III disease, further evidence is needed to establish their degree of benefit in this population.

First draft submitted: 3 March 2019; Accepted for publication: 13 June 2019; Published online:

2 September 2019

Keywords: immunotherapy • in-transit • melanoma • targeted therapies $\bullet$ unresectable

The management of metastatic melanoma has undergone a rapid transformation in recent years. The prognosis of patients with metastatic disease was historically poor, with a five-year overall survival (OS) of approximately $7 \%$ [1]. However, the advent of immune checkpoint inhibitors as well as BRAF and MEK inhibitors (for patients with $B R A F$-mutant melanoma) has dramatically transformed the therapeutic landscape. Immune checkpoint inhibitors have produced sustained improvements in OS, with 4-year OS for pembrolizumab of 37\% and the combination of ipilimumab and nivolumab producing a 3-year OS rate of 58\% [2,3]. Similarly, BRAF and MEK inhibition has demonstrated striking response rates and prolonged OS, with 3-year OS rates of 44\% for dabrafenib and trametinib [4]. However, there is limited data regarding the efficacy of these novel systemic therapies in those with unresectable locoregional disease, including unresectable in-transit metastases (ITM) and unresectable nodal disease.

Locoregional disease is defined as melanoma occurring at the site of the primary lesion, regionally in the draining lymph node basin, or anywhere in between these two sites (such as ITM) with the exclusion of distant metastatic disease. The natural history of locoregional disease is highly variable. Survival outcomes are more favorable than for stage IV disease, with a 5-year OS of 93, 83, 69 and 32\% for stage IIIA, IIIB, IIIC and IIID disease, respectively [5]. However, a significant percentage of patients with unresectable disease experience high levels of morbidity from their disease and eventually develop disease progression with distant metastases [6,7]. While some remain relatively 
indolent with multiple locoregional recurrences over many years prior to the development of disease metastases, others develop distant metastases rapidly.

\section{Technically versus clinically unresectable locoregional disease}

Unresectable locoregional disease can be divided into technically unresectable and clinically unresectable. Technically unresectable disease refers to disease for which surgical resection is not possible without unacceptable functional impairment or risk of major adverse events. This includes those with macroscopically resectable, but high-volume or multifocal disease, where there is a high likelihood of residual micrometastatic disease after surgery. In contrast, clinically unresectable disease includes two main groups of patients. First, those who are not suitable surgical candidates due to competing comorbidities. Second, those patients with technically resectable disease but in whom the tempo of disease progression suggests surgical futility. This latter group is difficult to define and highlights the importance of multidisciplinary team input into decision making.

\section{Systemic therapies for unresectable locoregional disease}

Unresectable locoregional disease can be managed either with a locoregional approach, the introduction of systemic therapies, or a combination of the two strategies. Locoregional approaches are reviewed elsewhere in this series (Henderson et al.) and include topical therapies, intralesional injections, radiotherapy, laser ablation or isolated limb infusion. While the efficacy of these therapies is well established, many patients will eventually recur. Furthermore, locoregional therapies may be technically difficult or even impossible depending on the site of disease. For example, pelvic nodal disease or ITM of the trunk or head and neck region are not amenable to isolated limb infusions or isolated limb perfusion. Systemic therapies, including immune checkpoint inhibitors and BRAF-MEK inhibition in $B R A F$-mutant disease, are increasingly being used in these patients given the routine application in patients with metastatic disease. They have the potential to alter the natural history of this condition by preventing the development of distant metastases, while limiting the morbidity that is associated with certain locoregional approaches. The choice between a locoregional or systemic approach ultimately depends on an individualized approach considering such factors as patient age, comorbidities, previous therapies, as well as disease characteristics including site of disease, disease volume, rate of progression and molecular characteristics.

Systemic agents are now increasingly used in clinical practice in unresectable locoregional disease, but there remains limited data available regarding the efficacy of both immune checkpoint inhibitors and BRAF-MEK inhibitors this setting. Studies to date have demonstrated differential response rates to immunotherapy in various organ sites with the highest rates of response in lung metastases [8]. Several case studies have demonstrated durable responses in patients with ITM treated with immune checkpoint inhibitors in combination with locoregional therapies $[9,10]$. The combination of ILI and ipilimumab in a Phase II trial evaluating of 18 patients with stage IIIB-IV melanoma patients demonstrated a progression-free survival of $57 \%$ at one year [11]. Forty-four percent of patients treated with combination of intralesional T-VEC (talimogene laherparepvec vaccine) and ipilimumab in a Phase Ib study of 19 patients with untreated stage IIIB-IV melanoma had a durable response after 6 months and PFS was not reached [10]. However, large registration studies of immune checkpoint inhibitors and BRAF-MEK inhibitors did not prespecify these specific groups during recruitment, and where these were analyzed retrospectively, conclusions are limited due to small patient numbers $[2-4,12-17]$.

\section{Immune checkpoint inhibitors}

Single agent ipilimumab (an antibody against CTLA-4) was the first immunotherapy agent to demonstrate efficacy over conventional chemotherapy in metastatic melanoma. Robert et al. compared ipilimumab in combination with dacarbazine to dacarbazine with placebo in 502 patients with treatment naive, unresectable stage III or stage IV melanoma. 3-year OS in the ipilimumab/dacarbazine group was $20.8 \%$ compared with $12.2 \%$ in the dacarbazine and placebo group [12]. However, only 14 patients (3\%) had unresectable stage III disease, and OS outcomes were therefore not examined in this small subgroup. Similarly, Hodi et al. examined the efficacy of ipilimumab, glycoprotein 100 (gp100) peptide vaccine and ipilimumab with gp100 in 676 patients with previously treated unresectable stage III or stage IV melanoma [13]. 2-year OS for ipilimumab, gp100 and combination ipilimumab and gp100 were $23.5,13.7$ and $21.6 \%$, respectively. However, only ten patients $(2 \%)$ had unresectable stage III disease. Therefore, the efficacy of ipilimumab in unresectable locoregional melanoma specifically remains uncertain.

Similar issues exist with the data for anti-programmed cell death protein (PD)-1 therapies. In KEYNOTE 001, 655 patients with unresectable stage III or stage IV melanoma who were either naïve treatment or previously treated 


\begin{tabular}{|c|c|c|c|c|c|c|}
\hline Authors & Study name & Agents & $n$ & $\begin{array}{l}\text { Unresectable stage III, } n \\
(\%)\end{array}$ & $\begin{array}{l}\text { Landmark overall survival, } \\
\% \text { (interval) }\end{array}$ & Ref. \\
\hline Robert et al. & & $\begin{array}{l}\text { Ipilimumab/dacarbazine } \\
\text { Placebo/dacarbazine }\end{array}$ & 502 & $14(3)$ & 20.8 (3 year) & [12] \\
\hline Hodi et al. & & $\begin{array}{l}\text { Ipilimumab/gp100 } \\
\text { Ipilimumab gp100 }\end{array}$ & 676 & $10(2)$ & 21.6 ( 2 year) & [13] \\
\hline Robert et al. & KEYNOTE 001 & $\begin{array}{l}\text { Pembrolizumab (ipilimumab naïve) } \\
\text { Pembrolizumab (all) }\end{array}$ & 655 & $8(1)$ & 41 (5 year) & [2] \\
\hline Long et al. & KEYNOTE 006 & $\begin{array}{l}\text { Pembrolizumab } \\
\text { Ipilimumab }\end{array}$ & 834 & $30(4)$ & 44 (4 year) & [14] \\
\hline Wolchok et al. & CHECKMATE 067 & $\begin{array}{l}\text { Ipilimumab/nivolumab } \\
\text { Nivolumab } \\
\text { Ipilimumab }\end{array}$ & 945 & $\begin{array}{l}397(42) \\
(\mathrm{M} 0, \mathrm{M} 1 \mathrm{a} \text { or } \mathrm{M} 1 \mathrm{~b})\end{array}$ & 58 (3 year) & [3] \\
\hline Long et al. & COMBI-D & $\begin{array}{l}\text { Dabrafenib/trametinib } \\
\text { Dabrafenib/placebo }\end{array}$ & 423 & $15(4)$ & 44 (3 year) & [4] \\
\hline Robert et al. & COMBI-V & $\begin{array}{l}\text { Dabrafenib/trametinib } \\
\text { Vemurafenib }\end{array}$ & 704 & $40(6)$ & 45 (3 year) & [15] \\
\hline Ascierto et al. & COBRIM & $\begin{array}{l}\text { Vemurafenib/cobimetinibk } \\
\text { Vemurafenib/placebo }\end{array}$ & 495 & $34(7)$ & 48.3 ( 2 year) & [16] \\
\hline Drummer et al. & COLUMBUS & $\begin{array}{l}\text { Encorafenib/binimetinib Encorafenib } \\
\text { Vemurafenib }\end{array}$ & 577 & $26(5)$ & 57.6 (2 year) & [17] \\
\hline
\end{tabular}

with ipilimumab were treated with pembrolizumab. The 5 -year OS rate was $34 \%$ in all patients and $41 \%$ in naïve treatment patients [2]. Eight patients (1\%) had unresectable stage III disease [8]. Similarly, KEYNOTE 006 compared two regimes of pembrolizumab with ipilimumab in 834 patients with unresectable stage III or IV melanoma. The 4 -year OS rate in the pooled pembrolizumab arms was $44 \%$ compared with $36 \%$ in the ipilimumab arm [14]. Thirty patients had unresectable stage III disease (4\%) [18]. Finally, the CHECKMATE 067 trial compared ipilimumab, nivolumab and combination ipilimumab and nivolumab in 945 patients with naïve treatment, unresectable stage III or stage IV melanoma. The 3 -year OS was $58 \%$ in the ipilimumab and nivolumab group, $52 \%$ in the nivolumab group and $34 \%$ in the ipilimumab group [3]. However, the number of patients with unresectable stage III was not specifically reported (M0, M1a or M1b: 397 [42\%]).

\section{Targeted therapies}

Unresectable stage III disease was also minimally represented in the registration studies of BRAF-MEK inhibitors. The COMBI-D trial compared dabrafenib and trametinib with dabrafenib and placebo in 423 patients with $B R A F$ mutant, naïve treatment unresectable stage IIIC or stage IV melanoma [4]. The 5-year OS was $44 \%$ for dabrafenib and trametinib compared with $32 \%$ for dabrafenib alone. Only 15 patients (4\%) had stage III disease [4]. Similarly, COMBI-V compared dabrafenib and trametinib with vemurafenib in 704 patients with BRAF V600E- or V600K-mutant treatment naive, unresectable stage III or stage IV melanoma [15]. The 3-year OS was $45 \%$ for dabrafenib and trametinib compared with $32 \%$ for vemurafenib [19]. Forty patients (6\%) had unresectable stage III disease [15]. CoBRIM compared vemurafenib and cobimetinib with vemurafenib and placebo in 495 patients with $B R A F$ mutant, treatment-naive, unresectable stage III or stage IV melanoma [16]. 2-year OS was $48.3 \%$ (41.4-55.2) in the vemurafenib and cobimetinib group and $38.0 \%$ (31.3-44.7) in the vemurafenib group [16]. Thirty-four patients (7\%) had unresectable stage III melanoma. Finally, COLUMBUS investigated encorafenib and binimetinib compared with encorafenib or vemurafenib alone in 577 patients with BRAF mutant, treatment-naive, unresectable stage III or stage IV melanoma [17]. The 2-year OS was 57.6\% in the encorafenib and binimetinib arm, $49.1 \%$ in the encorafenib arm and $43.2 \%$ in the vemurafenib arm [17]. Twenty-six patients (5\%) had stage IIIB/IIIC.

Therefore, despite the significant improvements in OSnoted for patients with metastatic disease, only a minority of patients in these studies had unresectable stage III disease $(<10 \%)$. In total, of the 4866 patients enrolled in seminal melanoma studies published over the last 5 years only 177 had stage III disease (3\%) (Table 1). The composition of the unresectable stage III groups was also not disclosed, making it difficult to draw conclusions 
about those with in-transit disease and unresectable nodal disease. Few studies characterized the number of sites or metastases, and did not specifically analyze these as predictors of response.

\section{Future perspective}

Further research is required to establish the utility of systemic therapies in unresectable locoregional melanoma. Prospective studies should prespecify unresectable locoregional disease as a subgroup for analysis, including those with in-transit disease and those with unresectable nodal disease. This will not only allow subgroup analyses of efficacy, but also help elucidate the clinicopathological predictors of response to systemic agents in unresectable stage III disease. Studies comparing locoregional therapies with systemic therapies would also be useful to guide practice, particularly in patients with technically unresectable disease due to possible residual micrometastases or clinically unresectable disease due to rapidly progressive disease. Optimal sequencing and combination strategies of locoregional and systemic therapies should also be examined. In the interim, retrospective data regarding the response rates and efficacy of systemic therapies in unresectable locoregional disease can assist in guiding practice.

\section{Conclusion}

Immune checkpoint inhibitors and BRAF-MEK inhibitors have redefined treatment paradigms in metastatic melanoma. Their potential to achieve durable responses and improve OS makes them a promising option earlier in the disease course in the context of unresectable locoregional melanoma. However, their efficacy and tolerability in this specific subgroup remains unknown due to their under-representation in seminal trials. Further research is required to determine the optimal timing for initiating these therapies, and their efficacy compared with and in conjunction with locoregional therapies.

\section{Executive summary}

\section{Context}

- Immunotherapy and targeted therapy produce sustained improvements in overall survival (OS) in metastatic melanoma.

- However, unresectable stage III disease is under-represented in seminal trials of novel therapies. Therefore, the application of this data to patients with unresectable stage III melanoma is unclear.

Definition of unresectable stage III disease

- Technically unresectable disease-where surgical resection is not possible without functional impairment or risk of major events or residual micrometastatic disease.

- Clinically unresectable disease-where surgery is contraindicated due to comorbidities or would be futile due to rapid disease progression.

Efficacy

- BRAF-MEK inhibitors (dabrafenib/trametinib) have a 3-year OS of $44 \%$ in patients with unresectable stage III and stage IV melanoma.

- Single agent anti-PD-1 (pembrolizumab) have a 4-year OS of $44 \%$ in patients with unresectable stage III and stage IV melanoma.

- Combination anti-CLTA-4 /anti-PD-1 (ipilimumab/nivolumab) have a three-year OS 58\% in patients with unresectable stage III and stage IV melanoma.

- Only three percent of patients enrolled in seminal melanoma studies published over the last 5 years had stage III disease.

Future directions

- Prospective studies should consider prespecifying subgroups of unresectable locoregional disease for analysis.

- Future directions include comparing systemic therapy with and in combination with locoregional therapies.

Conclusion

- Systemic agents are promising options earlier in the disease course of unresectable locoregional melanoma. However, their efficacy and tolerability in this specific subgroup remains unknown due to their under-representation in seminal trials. 


\section{Open access}

This work is licensed under the Attribution-NonCommercial-NoDerivatives 4.0 Unported License. To view a copy of this license, visit http://creativecommons.org/licenses/by-nc-nd/4.0/

\section{References}

Papers of special note have been highlighted as: $\bullet$ of interest; $\bullet \bullet$ of considerable interest

1. Balch CM, Gershenwald JE, Soong SJ et al. Final version of 2009 AJCC melanoma staging and classification. J. Clin. Oncol. 27(36), 6199-6206 (2009).

2. Robert C, Ribas A, Hamid O et al. Long-term outcomes in patients (Pts) with advanced melanoma treated with Pembrolizumab (Pembro): 4-year overall survival (OS) results from KEYNOTE-001. J. Clin. Oncol 35(15), 9504 (2017).

3. Wolchok JD, Chiarion-Sileni V, Gonzalez R et al. Overall survival with combined Nivolumab and Ipilimumab in advanced melanoma. N. Engl. J. Med. 377(14), 1345-1356 (2017).

- The first study to examine the combination of two immune checkpoint inhibitors (ipilimumab and nivolumab) and demonstrate an overall survival benefit compared with single agent ipilimumab.

4. Long GV, Flaherty KT, Stroyakovskiy D et al. Dabrafenib plus trametinib versus dabrafenib monotherapy in patients with metastatic BRAF V600E/K-mutant melanoma: long-term survival and safety analysis of a Phase III study. Ann. Oncol. 28(7), 1631-1639 (2017).

- The first study to demonstrate the efficacy of BRAF-MEK inhibition versus BRAF alone.

5. Gershenwald JE, Scolyer RA, Hess KR et al. Melanoma staging: evidence-based changes in the American Joint Committee on Cancer eighth edition cancer staging manual. CA. Cancer J. Clin. 67(6), 472-492 (2017).

6. Pawlik TM, Ross MI, Johnson MM et al. Predictors and natural history of in-transit melanoma after sentinel lymphadenectomy. Ann. Surg. Oncol. 12(8), 587-596 (2005).

7. Read RL, Haydu L, Saw RP et al. In-transit melanoma metastases: incidence, prognosis, and the role of lymphadenectomy. Ann. Surg. Oncol. 22(2), 475-481 (2015).

8. Ribas A, Hamid O, Daud A et al. Association of pembrolizumab with tumor response and survival among patients with advanced melanoma. JAMA 315(15), 1600-1609 (2016).

9. Ariyan CE, Lefkowitz RA, Panageas K et al. Safety and clinical activity of combining systemic ipilimumab with isolated limb infusion. $J$. Clin. Oncol. 32(15), 9078 (2014).

10. Puzanov I, Milhem MM, Minor D et al. Talimogene laherparepvec in combination with ipilimumab in previously untreated, unresectable Stage IIIB-IV melanoma. J. Clin. Oncol. 34(22), 2619-2626 (2016).

11. Ariyan CE, Lefkowitz RA, Panageas $\mathrm{K}$ et al. Safety and clinical activity of combining systemic ipilimumab with isolated limb infusion. $J$. Clin. Oncol. 32, 9078 (2014).

- One of the few prospective studies examining the efficacy of combining systemic and locoregional therapies.

12. Robert C, Thomas L, Bondarenko I et al. Ipilimumab plus dacarbazine for previously untreated metastatic melanoma. $N$. Engl. J. Med. 364(26), 2517-2526 (2011).

-. The first trial to demonstrate an overall survival benefit for immune checkpoint inhibitors (ipilimumab) in comparison with chemotherapy.

13. Hodi FS, O'day SJ, Mcdermott DF et al. Improved survival with ipilimumab in patients with metastatic melanoma. $N$. Engl. J. Med. 363(8), 711-723 (2010).

14. Long GV, Schachter J, Ribas A et al. 4-year survival and outcomes after cessation of pembrolizumab after 2-years in patients (pts) with ipilimumab (ipi)-naive advanced melanoma in KEYNOTE-006. J. Clin. Oncol. 36(15), 9503 (2018).

15. Robert C, Karaszewska B, Schachter J et al. Improved overall survival in melanoma with combined dabrafenib and trametinib. N. Engl. J. Med. 372(1), 30-39 (2015).

16. Ascierto PA, Mcarthur GA, Dreno B et al. Cobimetinib combined with vemurafenib in advanced BRAF(V600)-mutant melanoma (coBRIM): updated efficacy results from a randomised, double-blind, Phase III trial. Lancet Oncol. 17(9), 1248-1260 (2016).

17. Dummer R, Ascierto PA, Gogas HJ et al. Overall survival in patients with BRAF-mutant melanoma receiving encorafenib plus binimetinib versus vemurafenib or encorafenib (COLUMBUS): a multicentre, open-label, randomised, Phase III trial. Lancet Oncol. 19(10), 1315-1327 (2018).

18. Schachter J, Ribas A, Long GV et al. Pembrolizumab versus ipilimumab for advanced melanoma: final overall survival results of a multicentre, randomised, open-label Phase III study (KEYNOTE-006). Lancet 390(10105), 1853-1862 (2017).

19. Robert C, Karaszewska B, Schachter J et al. Three-year estimate of overall survival in COMBI-v, a randomized Phase III study evaluating first-line dabrafenib (D) + trametinib (T) in patients (pts) with unresectable or metastatic BRAF V600E/K-mutant cutaneous melanoma. Ann. Oncol. 27(Suppl. 6), LBA40-LBA40 (2016). 